\title{
TAXONOMIC NOVELTIES AND SYNOPSIS OF THE GENUS DIGITARIA (POACEAE, PANICOIDEAE, PANICEAE) IN PUERTO RICO AND THE VIRGIN ISLANDS
}

\author{
Andrea S. Vega ${ }^{1,4}$, Zulma E. Rúgolo de Agrasar ${ }^{2,4}$ \& Franklin S. Axelrod ${ }^{3}$
}

\begin{abstract}
${ }^{1}$ Universidad de Buenos Aires, Facultad de Agronomía, Departamento de Recursos Naturales y Ambiente, Cátedra de Botánica General, Av. San Martín 4453, C1417DSE, Buenos Aires, Argentina; avega@agro.uba.ar (author for correspondence).

${ }^{2}$ Instituto de Botánica Darwinion, Labardén 200, Casilla de correo 22, B1642HYD, Buenos Aires, Argentina.

${ }^{3}$ Herbario del Departamento de Biología, Facultad de Ciencias Naturales, Universidad de Puerto Rico - Río Piedras, PO Box 23360, San Juan, Puerto Rico.

${ }^{4}$ Members of Carrera del Investigador Científico, Consejo Nacional de Investigaciones Científicas y Técnicas (CONICET), Argentina.
\end{abstract}

\begin{abstract}
Vega, A. S., Z. E. Rúgolo de Agrasar \& F. S. Axelrod. 2018. Taxonomic novelties and synopsis of the genus Digitaria (Poaceae, Panicoideae, Paniceae) in Puerto Rico and the Virgin Islands. Darwiniana, nueva serie 6(2): 164-178.

The present paper reports 18 taxa of Digitaria in Puerto Rico and the Virgin Islands as a contribution to an ongoing floristic project. Digitaria filiformis var. dolichophylla, D. fragilis, D. sacchariflora, and $D$. similis constitute new reports. Digitaria ekmanii, D. fuscescens, D. panicea, and Digitaria $\times$ umfolozi are excluded taxa. This contribution includes synonymy, iconography, geographic distribution and habitat, vernacular names, specimens examined and a key for the identification of taxa. Digitaria filiformis var. dolichophylla is fully illustrated for the first time.
\end{abstract}

Keywords. Digitaria; Puerto Rico; taxonomy; Virgin Islands.

Resumen. Vega, A. S., Z. E. Rúgolo de Agrasar \& F. S. Axelrod. 2018. Novedades taxonómicas y sinopsis del género Digitaria (Poaceae, Panicoideae, Paniceae) en Puerto Rico e Islas Vírgenes. Darwiniana, nueva serie 6(2): 164-178.

El presente trabajo reporta 18 taxones de Digitaria en Puerto Rico e Islas Vírgenes, como contribución a un proyecto florístico en ejecución. Digitaria filiformis var. dolichophylla, D. fragilis, $D$. sacchariflora y $D$. similis constituyen nuevas citas. Digitaria ekmanii, $D$. fuscescens, $D$. panicea y Digitaria $\times$ umfolozi son taxones excluidos. Esta contribución incluye sinonimia, iconografía, distribución geográfica y hábitat, nombres vernáculos, exsicatta y una clave para la identificación de los taxones. Digitaria filiformis var. dolichophylla se ilustra en forma completa por primera vez.

Palabras clave. Digitaria; Islas Vírgenes; Puerto Rico; taxonomía.

\section{INTRODUCTION}

The genus Digitaria Haller emend. A.S. Vega \& Rúgolo (Vega \& Rúgolo de Agrasar, 2001) includes 271 species mainly in warm regions of both hemispheres (Soreng et al., 2017).
It includes foraging species, minor cereals, turf plants, and soil binders, as well as ornamental grasses (Rúgolo de Agrasar \& Puglia, 2004) and some important weeds. About 124 taxa occur in the New World (Vega \& Rúgolo de Agrasar, 2003), treated in partial taxonomic 
contributions (Rúgolo de Agrasar, 1974, 1994; Pohl, 1980; Renvoize, 1998; Canto-Dorow \& Longhi-Wagner, 2001; Giraldo-Cañas, 2005, 2016; Sánchez-Ken, 2012; Canto-Dorow, 2014; Vega \& Rúgolo de Agrasar, 2001, 2002, 2003, 2005, 2006, 2007, 2009, 2012, 2014; Renvoize et al., 2006; Lo Medico et al., 2017). A historical review of taxonomic studies started in the West Indies, where Hitchcock (1936) reported 16 species of Digitaria: D. argillacea (Hitchc. \& Chase) Fernald, D. argyrostachya (Steud.) Fernald, D. curvinervis (Hack.) Fernald, D. eggersii (Hack.) Henrard, D. ekmanii Hitchc., D. horizontalis Willd., D. insularis (L.) Fedde, D. ischaemum (Schreb.) Muhl., D. laxa (Rchb.) Parodi, D. longiflora (Retz.) Pers., D. panicea (Sw.) Urb., D. pinetorum Hitchc., D. sanguinalis (L.) Scop., D. serotina (Walter) Michx., D. villosa (Walter) Pers., and D. violascens Link, some of them previously considered under Trichachne Nees. Afterwards, Gould in Howard (1979) reported 10 species from the Lesser Antilles: Digitaria bicornis (Lam.) Roem. \& Schult., D. ciliaris, $D$. horizontalis, $D$. insularis, $D$. ischaemum, $D$. laxa, D. longiflora, D. sanguinalis, D. similis Beetle ex Gould, and D. violascens. In 2003, Vega and Rúgolo de Agrasar catalogued 21 species for the Caribbean and, specifically, 16 species were reported for Puerto Rico in
Axelrod (2011) and in the UPRRP database project (2018), but the lack of a synopsis of the genus for Puerto Rico and the Virgin Islands merits the present study.

The present paper reports 18 taxa of Digitaria in Puerto Rico and the Virgin Islands as a contribution to an ongoing floristic project (Peterson, unpubl.). Digitaria ekmanii, D. fuscescens, D. panicea and Digitaria $\times$ umfolozi are excluded taxa.

\section{MATERIALS AND METHODS}

Morphological studies were based on herbarium specimens housed at BAA, BM, C, G, GH, K, L, LP, MO, MVFA, NY, P, PRE, SI, SJ, UPRRP, US, VINPS, and W herbaria (acronyms after Thiers, 2018).

Digitaria Haller, Hist. Strip. Helv. 2: 244. 1768; emend. A.S. Vega \& Rúgolo, Amer. J. Bot. 88 (9): 1671. 2001.

Valota Adans., Fam. Pl. 2: 495. 1763.

Syntherisma Walter, Fl. Carol. 76. 1788.

Trichachne Nees, Fl. Bras. Enum. Pl. 2: 85. 1829.

Megaloprotachne C.E. Hubb., Bull. Misc. Inform. Kew 1929: 320-321. 1929.

\section{Key to the species of Digitaria in Puerto Rico and Virgin Islands}

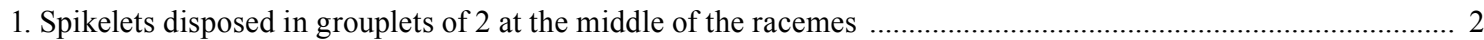

1. Spikelets disposed in grouplets of 3-10 at the middle of the racemes

2 (1). Spikelets with distant bracts, separated by conspicuous internodes. Spikelets pilose with lanose and acuminate hairs up to $6 \mathrm{~mm}$, exceeding the length of the spikelet. Indumentum whitish, tawny or ocraceous, largely exceeding the length of the spikelet. Perennials, caespitose, not stoloniferous

2. Spikelets with approximate bracts, separated by short internodes. Indumentum varied, whitish or brownish hairs at maturity, never lanose. Annuals or perennials, caespitose or stoloniferous

3 (2). Lower lemma 5 or 7-nerved, exposing the central 5 equally spaced nerves on the back .............................. 4

3. Lower lemma 7-nerved, exposing the central 3 nerves on the back, evenly spaced or not ................................... 6

4 (3). Lower lemma 7-nerved, glabrous on the back and pilose along the margins. Indumentum of the spikelets ochraceous D. similis

4. Lower lemma 5 or 7-nerved, glabrous on both sides of the midnerve and alternately pilose and glabrous in the remaining zones, or pilose between the nerves and along the margins. Indumentum of the spikelets silvery-whitish with purplish tints 
5. Lower lemma 5-nerved, with prominent nerves, densely pilose between the nerves and along the margin. Spikelets ellipsoid, 2.5-2.7 mm. Leaf blades adaxially shortly pubescent, abaxially glabrous. Ligule $0.2-0.5 \mathrm{~mm}$, truncate, margin ciliate; ligular zone glabrous or with sparse hairs

D. hitchcockii

5. Lower lemma 7-nerved, with scarcely visible nerves, glabrous on both sides of the midnerve and alternately pilose and glabrous in the remaining zones. Spikelets lanceolate, $3.7-4 \mathrm{~mm}$. Leaf blades pubescent on both surfaces. Ligule 1-4 mm, triangular or obtuse, margins erose; ligular zone hirsute

D. eggersii

6 (3). Panicle with 20-50 racemes, the distal portion pendulous. Plants $0.8-2.5 \mathrm{~m}$, generally taller than $1 \mathrm{~m}$. Leaf sheaths pilose. Leaf blades 1-2 $\mathrm{cm}$ wide. Lower glume acute

D. insularis

6. Panicle with 4-15 racemes, the distal portion straight. Plants 0.3-1 m. Leaf sheaths glabrous or sparsely pilose. Leaf blades $0.15-0.8(-1) \mathrm{cm}$ wide. Lower glume obtuse D. sacchariflora

7 (2). Spikelets usually heteromorphous. Subsessile spikelet with lower lemma glabrous or glabrous on both sides of the midnerve and alternately pilose and glabrous in the remaining zones. Pedicellate spikelet glabrous on both sides of the midnerve and alternately pilose and glabrous in the remaining zones with long translucent to whitish setiform hairs. If homomorphous, both spikelets with the same characters of the pedicellate spikelets. Lower glume apex obtuse

D. bicornis

7. Spikelets homomorphous, pilose, without setiform hairs. Lower lemma glabrous on both sides of the midnerve and alternately pilose and glabrous in the remaining zones. Lower glume triangular or truncate .................................... 8

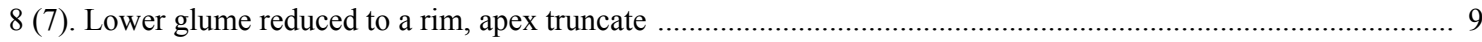

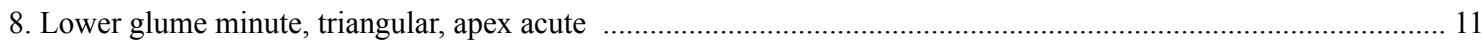

9 (8). Rachis winged, flat. Spikelets (2.7-)3-3.4 $\times 0.7-0.8 \mathrm{~mm}$. Upper glume $1 / 4^{-1 / 3}$ the length of the spikelet. Lower lemma with the midnerve distant from the lateral ones .......................................................................... D. setigera

9. Rachis not winged. Spikelets 1.8-2.2(-2.5) $\times 0.4-0.6 \mathrm{~mm}$. Upper glume $1 / 22^{2} / 3$ the length of the spikelet. Lower lemma with all nerves equally spaced 10

10 (9). Rachis with long hairs at the insertion of pedicels and racemes. Leaf sheaths pilose; ligular zone hirsute; leaf blades glabrous or pilose. Panicle 5-12 cm, with 4-7(-12) racemes on an axis 3-4 cm long. Upper glume $2 / 3-3 / 4$ the length of the spikelet

D. horizontalis

10. Rachis without long hairs at the insertion of pedicels and racemes. Leaf sheaths and blades glabrous; ligular zone glabrous, except with one or few hairs. Panicle 4-9.5 cm long, with 3-8 racemes on an axis up to $2.5 \mathrm{~cm}$ long. Upper glume $1 / 2$ the length of the spikelet

D. nuda

11 (8). Perennials, 0.6-1.2 m tall, culms erect. Leaf blades glabrous. Panicle up to $18 \mathrm{~cm}$ long with 3-14 racemes. Spikelets (2.5-)3-3.5 × ca. $1 \mathrm{~mm}$

D. eriantha

11. Annuals, $0.2-0.8 \mathrm{~m}$ tall, culms decumbent. Leaf blades hirsute. Panicle $8-10 \mathrm{~cm}$ long with 3-8 racemes. Spikelets (2.8-)3.2-3.5 × 0.7-0.8 mm D. ciliaris

12 (1). Rachis winged, flat, with conspicuous margins, wider than the midnerve ……........................................ 13

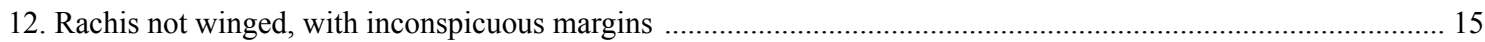

13 (12). Spikelets 2-2.5 $\times 0.8-1 \mathrm{~mm}$, with linear hairs with claviform apex and smooth walls .............. D. ischaemum

13. Spikelets 1.2-1.6 $\times$ 0.5-0.7 mm, with linear hairs with acute or obtuse apices and verrucose walls .................... 14

14 (13). Spikelets 1.5-1.6 mm long. Upper floret dark brown or purple-blackish at maturity. Anthers 0.4-0.6 mm long. Plants caespitose, erect, sometimes rooting at the lower nodes

D. violascens

14. Spikelets 1.2-1.3(-1.5) mm long. Upper floret yellowish or stramineous at maturity. Anthers 0.7-0.8 mm long. Plants stoloniferous

D. longiflora

15 (12). Rachis of the racemes hirsute, densely hirsute at the insertion of the racemes and pedicels. Pedicels hirsute D. fragilis

15. Rachis and pedicels scabrous 16

16 (15). Spikelets with linear hairs with a dilated and mucronate apex mixed with long, yellowish, setiform hairs D. argillacea var. argillacea

16. Spikelets with linear hairs with a claviform apex, without setiform hairs 17

17(16). Perennial. Leaf blades hirsute. Spikelets $2-2.5 \mathrm{~mm}$ long

17. Annual. Leaf blades glabrous. Spikelets $1.6-1.7 \mathrm{~mm}$ long D. villosa D. filiformis var. dolichophylla 
Digitaria argillacea (Hitchc. \& Chase) Fernald, Rhodora 22 (258): 104. 1920. Syntherisma argillaceum Hitchc. \& Chase, Contr. U.S. Natl. Herb. 18 (7): 296. 1917. TYPE: Puerto Rico, Monte Alegrillo, vicinity of Maricao, 2500 ft., 20-X-1913, A. Chase 6221 (holotype US!; isotypes, L, NY digital image!).

Iconography. Henrard (1950: 45); Vega \& Rúgolo de Agrasar (2005: 238).

Distribution and habitat. Caribbean, Mexico, Central America (Belize, Costa Rica, Guatemala, Honduras, Nicaragua, Panama), and South America (Colombia, Ecuador, Peru, Venezuela). Grows from sea level to $2100 \mathrm{~m}$ on grassy hills and slopes with scattered shrubs.

\section{Distribution in Puerto Rico and the Virgin Islands. Cerro Las Mesas, Mayagüez.}

Observations. $f l \& f r$ Oct. This species was cited by Britton \& Wilson (1923) as occurring at Monte Alegrillo near Maricao, at $800 \mathrm{~m}$ elevation, and at Monte Mesa, and subsequently cited by Liogier \& Martorell $(1982,2000)$, who clarify the former location as Cerro Las Mesas, Mayagüez.

\section{Additional specimens examined}

PUERTO RICO. Mayagüez. Dry rocky hill top between Rosario trail and Limon road, Monte Mesa, Chase 6271, 6277 (US).

Digitaria bicornis (Lam.) Roem. \& Schult., Syst. Veg. 2: 470. 1817. Paspalum bicorne Lam., Encycl. 1: 176. 1791. TYPE: "l' Inde", P. Sonnerat s.n. (holotype P-LAM; isotype US!, fragm.).

References. For complete synonymy see Veldkamp (1973: 30).

Iconography. Henrard (1950: 73); Vega \& Rúgolo de Agrasar (2012: 291).

Distribution and habitat. Introduced in America in tropical and subtropical regions. Extensively distributed from USA, the Caribbean,
Central America, and South America (Colombia, Venezuela, Guyana, Surinam, Brazil, Ecuador, Peru, Bolivia, Paraguay, and Argentina). Grows from sea level to $1500 \mathrm{~m}$, in disturbed areas, dry riverbeds, eroded slopes with naked soil and semideciduous forests.

Distribution and habitat in Puerto Rico and the Virgin Islands. Cabo Rojo, Fajardo, Juana Díaz, Lajas, Loíza, Mayagüez, Río Grande, Anegada, Guana Island, and Great Camanoe Island. 1-301 m.

Observations. $f l \& f r$ Jun-Apr.

\section{Additional specimens examined}

ANEGADA. Vicinit y of Set $t$ ing Point, Proctor 43666 (SJ).

GREAT CAMANOE ISLAND. Along ridge road, $\mathrm{S}$, portions island, 250-350 ft., in disturbed soil of clearings, Proctor 46541 (SJ).

GUANA ISLAND. Wit he Bay, Proctor 43881 (SJ).

PUERTO RICO. Cabo Rojo. Barrio Llanos Costa, ca. $1 \mathrm{~km}$ due ENE of Punta Molino, 2-10 $\mathrm{m}$, Proctor \& McKenzie 44952 (US). Fajardo. Cabezas de San Juan Nature Reserve, Axelrod \& Roubik 3877 (UPRRP, US). Juana Díaz. Sargent 3210 (US); Sierra Bermeja, Barrio Llanos Costa, summit of Cerro Mariquita, 250-301 m, Proctor 44365 (SJ); National Wildlife Refuge, 5-15 m, Proctor \& McKenzie 43817 (SJ). Lajas. Laguna Cartagena, Gould \& Liogier 15844 (US). Loíza. Barrio Torrecilla Baja, ridge between Punta Vacia Talega and Arenas village, 1-17.9 m, Proctor 49527 (SJ). Mayagüez. Isla de Mona, E side of isla, between the lighthouse and Cueva de Frío, 55-65 m, Proctor 43117 (SJ). Río Grande. Barrio Zarzal, beyond end of road 968, $\mathrm{km} \mathrm{4,} \mathrm{15-50} \mathrm{m,} \mathrm{E}$ and $\mathrm{N}$ of Cerro Bravo, Proctor 42670 (SJ).

Digitaria ciliaris (Retz.) Koeler, Descr. Gram.: 27. 1802. Panicum ciliare Retz., Observ. Bot. 4: 16. 1786. Panicum sanguinale L. var. ciliare (Retz.) Vasey, U.S. Dep. Agr. Div. Bot. Bul. 8: 23. 1889, nom. inval. TYPE: China, H. Wennerberg s.n. (lectotype LD, designated by Blake, Roy. Soc. Queensland 81: 11. 1969, again superfluously by Veldkamp, Blumea 21: 32. 1973). 
Panicum adscendens Kunth, Nov. Gen. Sp. (quarto ed.) 1: 97.1815 [1816]. Digitaria adscendens (Kunth) Henrard, Blumea 1: 92. 1934. TYPE: Mexico, in Nova Hispania juxta Zelaya et Querétaro, alt. 950 hexap., floret Septembri et Octobri, F. W. H. A. Humboldt \& A. J. A. Bonpland s.n. (lectotype P, designated by Veldkamp, Blumea 21: 32. 1973).

Digitaria tarapacana Phil., Verz. Antofagasta Pfl.: 80. 1891. TYPE: Chile, Pica, III-1885, F. Philippi s.n. (holotype SGO; isotypes US fragm. ex SGO, W!).

Iconography. Henrard (1950: 132); Vega \& Rúgolo de Agrasar (2012: 295).

Distribution and habitat. Digitaria ciliaris is one of the most frequent species in tropical and subtropical regions of both hemispheres, ranging from USA, the Caribbean, Central America, and South America (Venezuela, French Guiana, Surinam, Guyana, Brazil, Peru, Bolivia, Paraguay, Uruguay, Chile, and Argentina); also present in the Old World.

Distribution and habitat in Puerto Rico and the Virgin Islands. Adjuntas, Bayamón, Cabo Rojo, Carolina, Cataño, Coamo, Guánica, Guayama, Gurabo, Loíza, Mayagüez, Quebradillas, San Germán, San Juan, Trujillo Alto, Utuado, Vieques, Guana Island, St. Croix, St. Thomas and Virgin Gorda. 1-175 m.

Observations. $f l \& f r$ Jun-Apr.

\section{Additional specimens examined}

GUANA ISLAND. Vicinity of Guana Island

Club, 200 ft., Proctor 42507 (SJ).

PUERTO RICO. Adjuntas. Adjuntas Road, Chase 6481 (US). Bayamón. Heller \& Heller 4422 (US). Cabo Rojo. Boquerón, Chase 6510 $1 / 2$ (US); Barrio Llanos Costa, Cabo Rojo Nat. Wildlife Refuge, 5-15 m, Proctor \& Mckenzie 43817 (US); Proctor \& Mckenzie 43739 (SJ). Carolina. Barrio Sabana Abajo, just $\mathrm{N}$ of Campo Rico, Ave. at Sabana Gardens, $2 \mathrm{~m}$, Proctor et al. 46769 (SJ). Cataño. Cataño, Heller \& Heller 107 (US). Coamo. Vicinity of Coamo Spring, Río Coamo, Chase 6548 (US);
Coamo, Sintenis 3111 (US). Guánica. Road 333, km 3.1, playa Jaboncillo, Proctor 43368 (SJ). Guayama. Guayama, Chase 6755 (US). Gurabo. Barrio Jaguas, $1.3 \mathrm{~km}$ due NNW of Escuela Jaguas, on farm of L. Laing, elev. 150-175 m, Proctor 42933 (SJ). Loíza. Barrio Torrecilla Baja, between Punta Vacia Talega and Arenas village, 1-17.9 m, Proctor et al. 49521 (SJ). Mayagüez. Vicinity of Mayagüez, Chase 6253 (US); Monte Mesa, vicinity of Mayagüez, Chase 6180 (US); Mayagüez, N. L. Britton 2369 (US). Quebradillas. Barrio San José, along road following old railroad right-of-way, $07 \mathrm{~km} \mathrm{~W}$ of intersection with Road 4485, 40 m, Proctor 45918 (SJ). San Germán. Between San Germán and Sabana Grande, N. L. Britton et al. 4014 (US). San Juan. E of San Juan, Coco Beach at Río Grande on Atlantic Ocean, Gould et al. 15790 (US); Jardín Botánico, Univ. of Puerto Rico, Gould \& Martorell 15734 (US). Trujillo Alto. Chase 6364 (US). Utuado. Río Arecibo, between Utuado and Adjuntas, Chase 6460 (US). Vieques. North Coast, Chase 6670, 6688 (US).

ST. CROIX. St. Croix: West Indies Lab Compound, Teague Bay, Fosberg 53979 (US); "Crab Grass", Shocuprou 11 (US); Anna's Hope, Thompson 625 (US); Seashore, northside, Ricksecker 457 (US); Hitchcock 16331 (US).

ST. THOMAS. Neltjberg. At Neltjberg Bay, Proctor 45291, 45292 (SJ); Eggers 97, 232 (US).

VIRGIN GORDA. Valley, Fishlock 265 (US).

Digitaria eggersii (Hack.) Henrard, Blumea 1 (1): 97. 1934. Panicum eggersii Hack., Oesterr. Bot. Z. 51: 292. 1901. Valota eggersii (Hack.) Hitchc. \& Chase, Contr. U.S. Natl. Herb. 18 (7): 292. 1917. Trichachne eggersii (Hack.) Henrard, Meded. Rijks-Herb. 61: 10. 1930. TYPE: Virgin Islands, St. Thomas, Cowells Hill, 70 m, 1880, B. Eggers 295 (holotype W!; isotypes BR digital image!, K!, L digital image!, P!, US).

Iconography. Henrard (1950: 207); Lo Medico et al. (2017: 46).

Distribution and habitat. Caribbean. Grows on slopes near hills, from sea level to $300 \mathrm{~m}$. 
Distribution and habitat in Puerto Rico and the Virgin Islands. Cabo Rojo, Guana Island, St. Thomas and Virgin Gorda. 45-301 m.

Observations. $f l \& f r$ Oct-May.

\section{Additional specimens examined}

GUANA ISLAND. On W slope of Pyr amid Hill near Guana Head, Proctor 48822 (US); W slope of Pyramid Hill above Guana Head, 150-200 ft., Proctor 47251 (SJ); Proctor 48361 (SJ); Muskmelon Bay, 150 ft., Proctor $48814(\mathrm{SJ})$.

PUERTO RICO. Cabo Rojo. Sierra Bermeja, Barrio Llanos Costa, upper slopes and summit of Cerro Mariquita, 250-301 m, Proctor \& McKenzie 44125 (SJ).

ST. THOMAS. Cowell 's Hil l, Eggers s.n.(US); Insula Antillarum, St. Thomas, Eggers $3(\mathrm{~K})$.

VIRGIN GORDA. Valley, Fishlock 209 (US).

Digitaria eriantha Steud., Flora 12: 468. 1829. TYPE: South Africa. Cape: Cape of Good Hope, H. von Ludwig s.n. (holotype probably $\mathrm{P}$; isotype TUB digital image!).

Digitaria decumbens Stent, Bothalia 3 (1): 150. 1930. TYPE: South Africa: Transvaal: Barberton Dist., Nelspruit, 1928, J. A. Pentz 8495 (holotype PRE!; isotype US).

Digitaria pentzii Stent, Bothalia 3 (1): 147. 1930. TYPE: South Africa. Cape: Vrijburg, $J$. A. Pentz 8510 (holotype PRE!; isotype SRGH digital image!).

References. For complete synonymy see Kok (1984: 184).

Iconography. Rúgolo de Agrasar (1994: 97); Vega \& Rúgolo de Agrasar (2012: 296).

Distribution. Native of South Africa and introduced as a forage plant in Asia, Oceania, and America: USA, Mexico, the Caribbean, Central America (Guatemala, El Salvador, Honduras, Nicaragua, Costa Rica), and South America (Colombia, Venezuela, Brazil, Peru, Paraguay, Argentina).
Distribution and habitat in Puerto Rico and the Virgin Islands. Gurabo, Isabela, Manatí, Vega Baja and St. Croix. 0-800 m.

Observations. $f l \& f r$ Sep-Jan.

Additional specimens examined

PUERTO RICO. Gurabo. Barrio Jaguas, $1.3 \mathrm{~km}$ due NNW of Escuela Jaguas, on farm of L. Laing, 150-175 m, Proctor 42924 (US); disturbed area just $\mathrm{S}$ of La Tosca, S of Road 115, Km 4.6, sea level, Proctor 42908 (US). Isabela. Barrio Llanados, field near W bank of Río Guajataca, 2-3 km above Highway 2 bridge, 5-8 m, Proctor et al. 45909 (SJ). Manatí. Barrio Tierras Nuevas Salientes, 0.7$1.4 \mathrm{~km}$ E of Road 686 at Baldwin School, SE of El Pulguero, probably planted, Proctor 42211 (SJ). Vega Baja. Barrio Cabo Caribe, ca. $1 \mathrm{~km}$ due $\mathrm{W}$ of Central San Vicente, $5 \mathrm{~m}$, Proctor 44335 (SJ).

ST. CROIX. Northside. Maroon Ridge area NE, of Springgarden $800 \mathrm{~m}$, Proctor $44986(\mathrm{SJ})$.

Digitaria filiformis (L.) Koeler var. dolichophylla (Henrard) Wipff, Phytologia 80 (5): 348. (1996) 1997. Digitaria dolichophylla Henrard, Blumea 1 (1): 94. 1934. TYPE: USA, Florida, Dade Co., Buena Vista, 5-XII-1903, A. A. Eaton 459 (holotype $\mathrm{L}$; isotype US!). Fig. 1.

Iconography. Henrard (1950: 204).

Distribution. Caribbean and USA.

Distribution in Puerto Rico and the Virgin Islands. Manatí.

Observations. $f l \& f r$ Nov.

Additional specimens examined

PUERTO RICO. Manatí. Campo Alegre, between Manatí and Laguna del Tortuguero, Chase 6435 (US).

USA. Florida. Miami, roadside, A. Chase 3844 (US). 


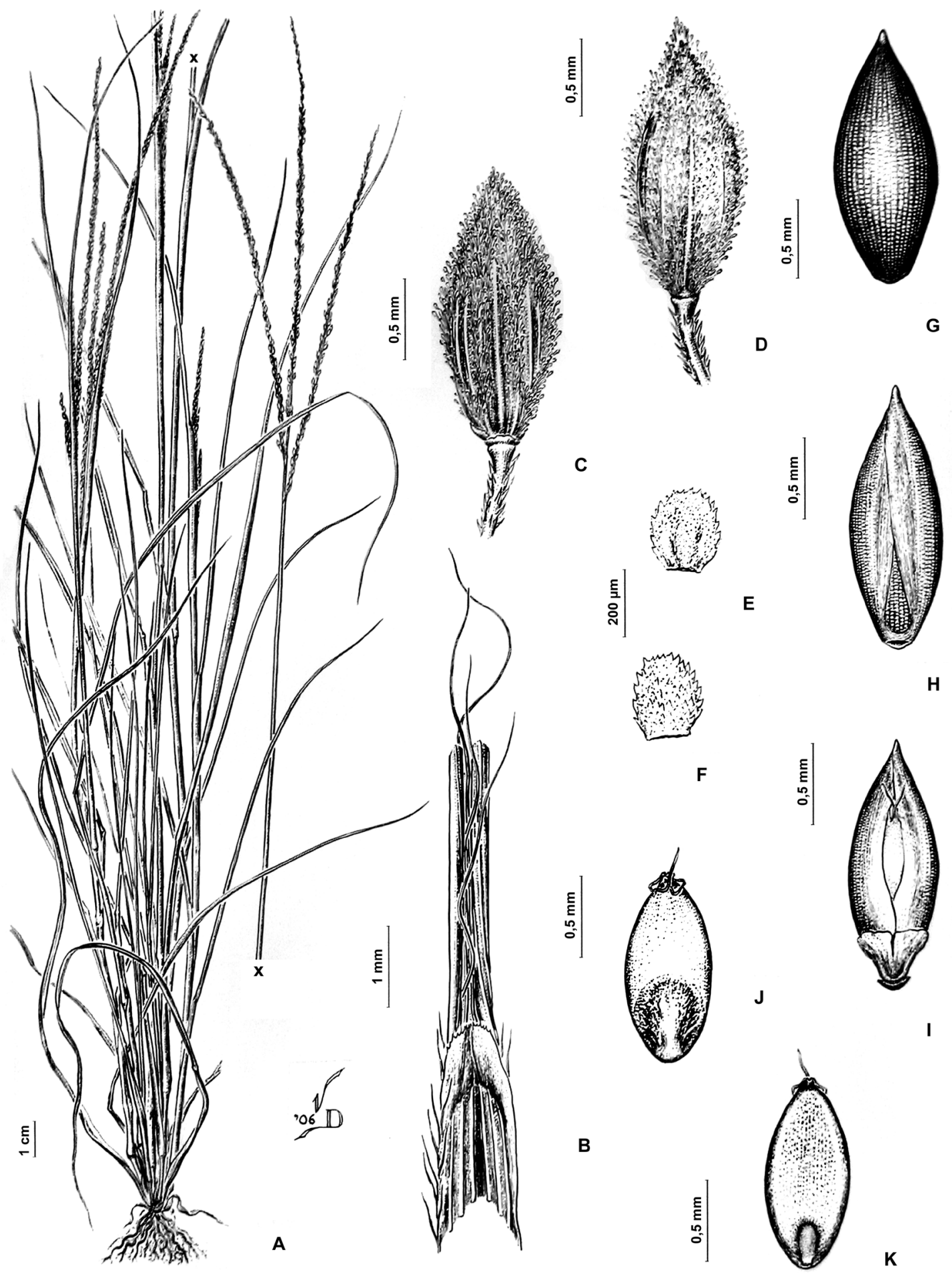

Fig. 1. Digitaria filiformis var. dolichophylla. A, habit. B, ligule. C, spikelet view from lower glume and lemma. D, spikelet view from the upper glume. E, lower palea, ventral view. F, lower palea, dorsal view. G, upper floret view from the back of the lemma. H, upper floret view from the back of the upper palea. I, lodicules, upper palea, and caryopsis. J, caryopsis, scutellar view. K, caryopsis, hilar view. A-K, from Chase 3844 (US). 
Digitaria fragilis (Steud.) Luces, J. Wash. Acad. Sci. 32 (6): 160. 1942. Paspalum fragile Steud., Syn. Pl. Glumac. 1: 17. 1853. TYPE: Venezuela, Cumaná, savanas de Guanaguana, 1843, N. Funck 724 (holotype P!; isotypes BAA fragm. ex P!, BM!, G!, P!, US fragm. ex P digital image!, $\mathrm{W}$ !).

Digitaria rhachitricha Henrard, Blumea 1 (1): 95. 1934. TYPE: Colombia, N. Funck \& L. Schlim 724 (holotype, L!; isotype $\mathrm{W}$ digital image!).

Iconography. Renvoize et al. (2006: 141).

Distribution. Caribbean, Central America (Nicaragua, Panama) and South America (Colombia, Venezuela, Guyana, Ecuador, Brazil, Bolivia).

Distribution and habitat in Puerto Rico and the Virgin Islands. Mayagüez. ca. $370 \mathrm{~m}$.

Observations. $f l \& f r$ Sep-Nov.

Additional specimens examined

PUERTO RICO. Mayagüez. Cerro Las Mesas, Barrio Rosario, Road 349, km. 6.6, ca. 370 m, Proctor \& McKenzie 43911 (SJ, US); Las Mesas, Torres 8 (US).

Digitaria hitchcockii (Chase) Stuck., Annuaire Conserv. Jard. Bot. Geneve 17: 287. 1914. Valota hitchcockii Chase, Proc. Biol. Soc. Washington 24: 110. 1911. Trichachne hitchcockii (Chase) Chase, J. Wash. Acad. Sci. 23 (10): 454. 1933. TYPE: USA, Texas, San Antonio, dry prairie soil, 18/24-VI-1910, A. S. Hitchcock 5329 (holotype US!; isotype BAA!).

Iconography. Henrard (1950: 326); Lo Medico et al. (2017: 47).

Distribution and habitat. USA, Mexico, and the Caribbean. Inhabits dry rocky slopes and sandy soils from 350-1600 m.

Distribution in Puerto Rico and the Virgin Islands. St. John.

Additional specimens examined

ST. JOHN. Ram Head Trail, Woodbury 189 (VINPS).
Digitaria horizontalis Willd., Enum. P1.: 92. 1809. Panicum horizontale (Willd.) G. Mey., Prim. Fl. Esseq.: 54. 1818. Panicum sanguinale L. var. horizontale (Willd.) Schweinf., Bull. Herb. Boissier 2, Appendix 2: 18. 1894. TYPE: Santo Domingo, Anon. (holotype B-W; isotypes MVFA!, US).

Iconography. Henrard (1950: 332); Vega \& Rúgolo de Agrasar (2012: 298).

Distribution. Throughout the tropics and subtropics. Extensively distributed in Africa, Asia, and America from USA to Argentina.

Distribution and habitat in Puerto Rico and the Virgin Islands. Cabo Rojo, Cataño, Cayey, Ciales, Coamo, Dorado, Fajardo, Loíza, Luquillo, Manatí, Mayagüez, Naguabo, Ponce, Salinas, San Juan, Vega Alta, Vieques, Mona, St. Croix, St. John, and St. Thomas. 0-830 m.

Observations. $f l \& f r$ throughout the year.

\section{Additional specimens examined}

MONA. In cultivated 1 and near coast, Hess 432 (US); Sardiñera, Britton et al. 1784 (US).

PUERTO RICO. Cabo Rojo. Barrio Boquerón, $\mathrm{N}$ end, Balneario Público de Boquerón, near sea level, Proctor 43760 (SJ). Cataño. Cataño, Millspaugh 333 (US). Cayey. Cayey, Sintenis 2473 (US). Ciales. Sargent 3059 (US). Coamo. Coamo Springs, along Río Coamo, Chase 6544 (US). Dorado. Barrio Higuillar, just E of Aeropuerto de Dorado, 8-10 m, Proctor 42450 (SJ). Fajardo. Fajardo, Sintenis 1255 (US); Barrio Quebrada Vueltas, ca. 2-3 km due SE of Fajardo town, 5-80 $\mathrm{m}$, Proctor \& Torres 50774 (SJ). Loíza. Barrio Torrecilla Baja, ridge between Punta Vacia Talega and Arenas village, 1-17.9 m, Proctor et al. 49505 (SJ); Road 187, ca. $0.5 \mathrm{~km} \mathrm{NW}$ of Río Grande de Loíza, near sea level, Proctor 42149 (SJ). Luquillo. Barrio Sabana, Otero 534 (US). Manatí. Campo Alegre, between Manatí and Laguna del Tortuguero, Chase 6619 (US). Mayagüez. $5 \mathrm{mi}$ NE of Mayagüez, Heller 4398 (US). Naguabo. Sierra de Naguabo, Loma Icaco, Shafer 3405 (US). Ponce. Ponce to Aibonito, Chase 6331 (US). Salinas. Barrio Lapa, summit area of east peak, 
Las Tetas de Cayey, 820-830 m, Proctor 42996 (SJ); Proctor \& McKenzie 44048 (SJ). San Juan. Río Piedras, Johnston 127 (US). Vega Alta. Barrio Sabana, silica-sand area NE of Regadera, $2.2 \mathrm{~km}$. Due NE of Intersection Roads 690 and 691, $5 \mathrm{~m}$, Proctor \& Thomas 45089 (SJ); Proctor 41854 (SJ). Vieques. vicinity of Isabel Segunda, Shafer 2469 (US); cliff north coast, Chase 6672 (US); Monte Pirata, N slope, along telephone lines and in forest bordering them, 200-225 m, Axelrod 4931 (UPRRP, US).

ST. CROIX. Bassin, Ricksecker 45 (US); Hitchcock 16348 (US).

ST. JOHN. Viers, Acevedo-Rodríguez \& Alemán 3211 (US).

ST. THOMAS. Eggers s.n. (NY, US); Hitchcock 16320 (US).

Digitaria insularis (L.) Fedde, Just's Bot. Jahresber. 31: 778. 1904. Andropogon insularis L., Syst. Nat. ed. 10, 2: 1304. 1759. Panicum insulare (L.) G. Mey., Prim. Fl. Esseq.: 60. 1818. Trichachne insularis (L.) Nees, Fl. Bras. Enum. Pl. 2: 86. 1829. Tricholaena insularis (L.) Griseb., Abh. Königl. Ges. Wiss. Göttingen 7: 265. 1857. Syntherisma insulare (L.) Millsp. $\&$ Chase, Publ. Field Columb. Mus., Bot. Ser. 1: 473. 1902. Valota insularis (L.) Chase, Proc. Biol. Soc. Wash. 19: 188. 1906. Digitaria insularis (L.) Mez ex Ekman, Ark. Bot. 11 (4): 17. 1912, comb. superfl. TYPE: Jamaica, $P$. Browne s. $n$. (lectotype LINN, designated by A. S. Hitchcock, Contr. U.S. Natl. Herb. 12: 126. 1908).

Panicum leucophaeum Kunth, Nov. Gen. Sp. (quarto ed.) 1: 97. 1815 [1816]. Panicum insulare var. leucophaeum (Kunth) Kuntze, Revis. Gen. Pl. 3 (3): 361. 1898. Digitaria leucophaea (Kunth) Stapf, Fl. Cap. 7: 382. 1898. TYPE: Venezuela, Cumaná, A. Humboldt \& A. J. A. Bonpland s. $n$. (holotype P!; isotypes BAA!, P!, US).

Panicum saccharoides A. Rich., Hist. Fis. Cuba, Bot. 11: 306. 1850. TYPE: Cuba, $R$. de la Sagra 9 (holotype P!; isotype P!).

Panicum falsum Steud., Syn. Pl. Glumac. 1: 67. 1853. TYPE: "Ins. Cuba" (holotype P).
Panicum duchaissingii Steud., Syn. Pl. Glumac. 1: 93. 1853. TYPE: "Duchaissing legit in Guadaloupe" (holotype P!).

Iconography. Henrard (1950: 347); Rúgolo de Agrasar (1974: 88); Vega and Rúgolo de Agrasar (2012: 298).

Common name. "Long Grass" (Hitchcock 16299, US).

Distribution and habitat. Digitaria insularis was originally described for Jamaica and introduced in Asia and Oceania. It is frequent in varied habitats as a weed in tropical and subtropical crops, also along the roadsides and sandy soils from sea level to $1900 \mathrm{~m}$. It ranges from USA to Argentina.

Distribution and habitat in Puerto Rico and the Virgin Islands. Adjuntas, Aguada, Bayamón, Cayey, Ciales, Coamo, Culebra, Fajardo, Guánica, Gurabo, Maricao, Mayagüez, Morovis, Naguabo, Salinas, San Germán, San Juan, Utuado, Vega Baja, Vieques, Yauco, Desecheo, Guana Island, Saba, St. Croix, St. John, St. Thomas, Tortola, and Vieques Island. 1-925 m.

Observations. $f l \& f r$ throughout the year.

Additional specimens examined

DESECHEO. Hess 428 (US).

GUANA ISLAND. S. of Island Club, $100 \mathrm{~m}$, Proctor 43404 (SJ).

PUERTO RICO. Adjuntas. Adjuntas Road, 4 mi from Ponce, Heller 6158 (US). Aguada. Barrio Guaniquilla; SSE of Rd. 441, km 1.5, Proctor 47753 (SJ, US). Bayamón. SW of Bayamón, Blomquist 12060 (US); branch road 879, $1 \mathrm{~km}$ due WSW of Co. La Peña, 510-520 m, Proctor 43517 (SJ); Bayamón, Chase 6375 (US). Cayey. Monte Llano, Goll et al. 479 (US). Ciales. Johnston 972 (US). Coamo. Coamo Springs, Underwood \& Griggs 564 (US). Culebra. Britton \& Wheeler 48 (US). Fajardo. Barrio Quebrada Vueltas, Cerro Fajardo, 35-110 m, Proctor \& Gonzalez 50793 (SJ). Guánica. Ensenada, Guánica Bay, cleared limestone hillside, Chase 6525 (US). Gurabo. Barrio Jaguas, $1.3 \mathrm{~km}$ due NNW of Escuela Jaguas, on farm L. Laing, 150-175 m, Proctor 42923 (SJ). 
Maricao. Indiera Fría, vicinity of Maricao, Chase 6240 (US). Mayagüez. Guanajibo, vicinity of Mayagüez, Chase 6284 (US); Prope Mayaguez, Sintenis 26 (US); Road from Mayagüez, to Joyuda, Underwood \& Griggs 131 (US); Prope Maricao, Hacienda Piñán, Sintenis 213 (US). Morovis. Barrio Río Grande de Manatí, at Road 155, 130 m, Proctor 42296 (SJ). Naguabo. Barrio Húcares, East Fortune Hill, Freid \& Richey 99-181 (UPRRP). Salinas. Barrio Lapas, vicinity of Las Tetas de Cayey, 810-830 m, Proctor 42815 (SJ). San Germán. Campus of InterAmerican University in Santa Marta Hills outside San Germán, Stimson 1201 (US). San Juan. Río Piedras, 1 1/2 mi S of Military Road, Heller \& Heller 136 (US); Barrio Puerta de Tierra, along Ave. Ponce de León, 15 m, Proctor 45325 (SJ). Utuado. NW of Utuado, Blomquist 11808 (US). Vega Baja. Vega Baja, Heller \& Heller 650 (US). Vieques. North coast west of town, Chase 6684 (US). Yauco. Yauco \& Ponce, Chase 6328 (US).

SABA. Wells Bay road, 22 m, 20 Feb 2015, Axelrod et al. 15781 (UPRRP).

ST. CROIX. Green Cay, Fosberg 59283 (US); Hitchcock 16343 (US); Bassin, Ricksecker 396 (US).

ST. JOHN. Virgin Island. Reef Bay Quarter, along new road from Bordeaux Road to Coral Bay. 242 m, Acevedo-Rodríguez \& Siaca 3800 (US); Centerline Road at Junction to Bordeaux Road, 270 m, Acevedo-Rodríguez 2619 (UPRRP, US); Maho Bay Quarter, 3 m, Acevedo-Rodríguez et al. 1943 (US); Cruz Bay Quarter, east side of Gift Hill, Acevedo-Rodríguez 818 (UPRRP, US); Morrow 5 (US).

ST. THOMAS. Archer 2226 (US); Hitchcock 16299 (US); Eggers s.n. (US); Morrow 43 (US); Charlotte Amalie, Millspaugh 385, 539 (US).

TORTOLA. Harrigan Exp. Station, Fairchild 3863 (US).

VIEQUES ISLAND. Vicinity of Isabel Segunda, Shafer 2435 (US).

Digitaria ischaemum (Schreb.) Schreb. ex Muhl., Descr. Gram.: 131. 1817. Panicum ischaemum Schreb., Spec. Fl. Erlang. 1: 16. 1804. Syntherisma ischaemum (Schreb.) Nash, N. Amer. Fl. 17 (2): 151. 1912. TYPE: Germany, Bavaria (holotype M).
Digitaria humifusa Pers., Syn. Pl. 1: 85. 1805. TYPE: France, hab. in arenosis circa Parisios, A. Richard s.n.(lectotype L!, designated by Henrard 1950: 337).

Syntherisma glabrum Schrad., Fl. Germ. 1: 163. 1806. Panicum glabrum (Schrad.) Gaudin, Agrost. Helv. 1: 22. 1811. Digitaria glabra (Schrad.) P. Beauv., Ess. Agrostogr. 51. 1812. TYPE: In cultis et incultis, ad vias alibique (holotype non-localized).

Iconography. Rúgolo de Agrasar (1974: 129).

Distribution. Native to Europe and temperate Asia. Introduced in Oceania and America (Canada, USA, the Caribbean, and Chile).

Distribution and habitat in Puerto Rico and the Virgin Islands. Río Grande. Herb cited by Liogier \& Martorell $(1982,2000)$ as occurring in waste grounds at lower elevations.

\section{Observations. $f l \& f r$ Oct.}

\section{Additional specimens examined}

PUERTO RICO. Río Grande. Sierra de Luquillo, Caribbean National Forest, summit El Yunque, $1065 \mathrm{~m}$, on moist grassy roadside bank near end of road, Proctor 42279 (SJ).

Digitaria longiflora (Retz.) Pers., Syn. Pl. 1: 85. 1805. Paspalum longiflorum Retz. Observ. Bot. 4: 15. 1786. TYPE: India, K. D. Koenig s.n. (holotype LD; isotypes BM, C!, K).

References. For full synonymy, see Veldkamp (1973: 66).

Iconography. Henrard (1950: 410); Vega \& Rúgolo de Agrasar (2005: 258).

Distribution. Native to temperate and tropical regions of the Old World. Introduced in USA, Costa Rica, the Caribbean, Colombia, and Guyana.

Distribution and habitat in Puerto Rico and the Virgin Islands. San Juan and Vega Baja. 1-2 m. 
Observations. $f l \& f r$ May-Oct.

\section{Additional specimens examined}

PUERTO RICO. San Juan. Río Piedras, Alberts 274 (US). Vega Baja. Barrio Algarrobo, just S of Laguna Tortuguero, in wet silica sand, Proctor 45035 (SJ).

Digitaria nuda Schumach., Beskr. Guin. Pl.: 45. 1827. TYPE: Africa, Ghana, P. Thonning 367 (holotype C; isotype L!).

Iconography. Henrard (1950: 500); Rúgolo de Agrasar (1994: 121, Fig. 34, A-D).

Distribution. Native to Africa. Tropical regions of the world. Introduced in the New World: USA, Mexico, the Caribbean, and South America (Venezuela, Guyana, Surinam, French Guiana, Brazil, Bolivia, Paraguay, and Argentina).

Distribution and habitat in Puerto Rico and the Virgin Islands. Manatí, Quebradillas, Mona, and St. Thomas. 10-500 m.

Observations. $f l \& f r$ Feb-May.

\section{Additional specimens examined}

MONA. Hess 456 (US).

PUERTO RICO. Manatí. Western end of Laguna Tortuguero, Britton \& Chardon 6834 (US). Quebradillas. Vicinity of Quebradillas, Chase 6568 (US), Chase 6576 (US).

ST. THOMAS. Eggers s.n. (US).

Digitaria sacchariflora (Nees) Henrard, Blumea 1:99. 1934. Trichachne sacchariflora Nees, Fl. Bras. Enum. Pl. 2: 87. 1829. Panicum sacchariflorum (Nees) Steud., Syn. Pl. Glumac. 1: 97. 1854. Panicum insulare (L.) G. Mey. var. sacchariflorum (Nees) Hack., Anales Mus. Nac. Buenos Aires 11 (3): 71. 1904. TYPE: Brazil, Paraná, Ponta Grossa, camino a Tibagi, a $13 \mathrm{~km}$ de ruta BR-376, 12-XII-1992, Z. Rúgolo et al. 1691 (neotype SI! designated by Vega \& Rúgolo, Syst. Bot. 42: 48. 2017).
Leptocoryphium penicilligerum Speg., Anales Soc. Ci. Argent. 16: 102. 1883. Panicum penicilligerum (Speg.) Hack., Allg. Bot. Z. Syst. 9: 93. 1902. Panicum insulare var. penicilligerum (Speg.) Hack., Anales Mus. Nac. Buenos Aires 11: 70. 1904. Valota penicilligera (Speg.) Chase ex Parodi, Revista Fac. Agron. Veterin. (Buenos Aires) 4: 46. 1922. Trichachne penicilligera (Speg.) Parodi, Gram. Bonaer. (ed. 3): 86. 1939. TYPE: Argentina, "Chaco, prope coloniam Resistencia”, 20-IV-1883, C. Spegazzini s.n. (lectotype LP! designated as "type" by Veldkamp, Blumea 21: 49. 1973; isolectotype BAA!).

Iconography. Henrard (1950: 643); Rúgolo de Agrasar (1974: 88, Fig. 5 i-j).

Distribution and habitat. Central America (Guatemala, El Salvador), the Caribbean, and South America (Colombia, Venezuela, Brazil, Peru, Bolivia, Paraguay, Argentina, Uruguay). Frequent in sandy soils of subtropical regions and in river ravines from sea level to $2020 \mathrm{~m}$.

Distribution in Puerto Rico and the Virgin Islands. Mona Island, Guana Island, and St. Thomas.

Observations. $f l \& f r$ Oct-Feb.

\section{Additional specimens examined}

GUANA ISLAND. Along crest of ridge between Guana Island Club \& Muskmelon Bay, 150-260 ft., Proctor 46522 (SJ).

PUERTO RICO. Mona Island. In cultivated land near coast, Hess 433 (US).

ST. THOMAS. Charlotte Amalie, Millspaugh 368 (US); Eggers s.n. (US).

Digitaria setigera Roth, Syst. Veg. (ed. 15bis) 2: 474. 1817. TYPE: India, B. Heyne s.n. (holotype $\mathrm{B}$ !; isotype $\mathrm{K}$ ! fragm.).

References. For full synonymy, see Veldkamp (1973: 37).

Iconography. Renvoize et al. (2006: 150). 
Distribution. Native to tropical regions of the Old World. Digitaria setigera is naturalized in USA, Mexico, Central America, the Caribbean, and South America (Colombia, Guyana, Surinam, French Guiana, Ecuador, Peru, and Bolivia).

Distribution and habitat in Puerto Rico and the Virgin Islands. Cayey, Morovis, and San Lorenzo. 1-900 m.

Observations. $f l \& f r$ throughout the year. Specimens of this taxon have commonly been misidentified as Digitaria sanguinalis (L.) Scop.

\section{Additional specimens examined \\ PUERTO RICO. Cayey. Barrio Lapa, summit of E peak, Las Tetas de Cayey, 820-830 m, McKenzie 44064 (SJ); Carite Forest Reserve, along road to Cerra la Santa, Axelrod 7233 (US). Morovis. Barrio Nuevo, along ridge $\mathrm{W}$ of Cerro Avispa, 500-550 m, Proctor 42942 (SJ). San Lorenzo. Cerro La Santa, $800 \mathrm{~m}$, H. Liogier 37066 (MO).}

Digitaria similis Beetle ex Gould, J. Arnold Arbor. 60 (2): 320. 1979. Trichachne affinis Swallen, Rhodora 65: 355. 1963. TYPE: Dominican Republic, Santiago, Valle del Cibao, roadsides at Quinigua, 21-X-1930, E. L. Ekman s. n. (holotype US !; isotypes BAA!, BM!, GH!, SI!).

Digitaria affinis Opiz ex Bercht., Oekon.-techn. Fl. Böhm. 1: 500. 1836, hom. illeg., non Roem. \& Schult. 1817.

Iconography. Rúgolo de Agrasar (1992: 58); Vega \& Rúgolo de Agrasar (2012: 305).

Distribution and habitat. Central America, the Caribbean, and South America (Colombia, Venezuela, Brazil, Peru, Bolivia, Paraguay, and Argentina); occurs along roadsides in disturbed areas, in sandy clay soils and on shores of rivers and streams, from sea level to $2500 \mathrm{~m}$.

Distribution in Puerto Rico and the Virgin Islands. St. Thomas.
Additional specimens examined

ST. THOMAS. In agris vulgarisimis, II-1887, Eggers 890 (P); sine data, Duchaissing s.n. (P); 1841, Friedrichstal 229 (W).

Digitaria villosa (Walter) Pers., Syn. P1. 1: 85. 1805. Syntherisma villosum Walter, Fl. Carol. 77. 1788. TYPE: USA, T. Walter s.n. (holotype BM).

Syntherisma leucocoma Nash, Bull. Torrey Bot. Club 25: 295. 1898. TYPE: USA. Florida, Lake Co., 3-VII-1894, N. Nash 1155 (holotype NY; isotype US!).

Digitaria obtusa Swallen, Phytologia 4 (7): 425. 1953. TYPE: Guatemala. Alta Verapaz, VIII1912, H. von Tuerckheim 3794 (holotype US!).

References. For full synonymy, see Vega \& Rúgolo de Agrasar (2003: 213).

Iconography. Vega \& Rúgolo de Agrasar (2007: 109).

Distribution. USA, Mexico, Central America, and the Caribbean.

Distribution in Puerto Rico and the Virgin Islands. Lares, Manatí, Mayagüez, and Trujillo Alto.

Observations. $f l \& f r$ Dec-Feb.

Additional specimens examined

PUERTO RICO. Lares. Campo Alegre, between Manati and Laguna del Tortuguero, Chase 6806 (US). Manatí. Laguna Tortuguero, Britton et al. 3858 (US). Mayagüez. Monte Mesa, between Rosario trail and Limon road, Chase 6271 (US). Trujillo Alto. Near Lake Loisa, vicinity of San Juan, Chase 6784 (US).

Digitaria violascens Link, Hort. Berol. 1: 229. 1827. Panicum violascens (Link) Kunth, Révis. Gramin. 1: 33. 1829. Syntherisma violascens (Link) Nash, Proc. Acad. Nat. Sci. Philadelphia 61 (3): 488. 1909. TYPE: "Hb. in Brasilia" (holotype B; isotype BAA!). 
Paspalum fuscum J. Presl., Reliq. Haenk. 1: 214. 1830. TYPE: "Hab. in Luzonia? in Peruviae montanis huanoccensibus, 1891, T. Haenke 153" (holotype L!; isotype MO!).

Paspalum chinense Nees, in Hook. \& Arn., Bot. Beechey Voy. 231. 1841. Syntherisma chinensis (Nees) Hitchc., Contr. U. S. Natl. Herb. 22 (6): 468. 1922. TYPE: China, ad Macao urbem, $G$. H. Vachell p.p. (holotype CAM).

Paspalum minutiflorum Steud., Syn. Pl. Glumac. 1: 17. 1853. TYPE: "Japon" (holotype P).

Syntherisma helleri Nash, Minnesota Bot. Stud. 1: 798. 1897. TYPE: USA. Hawaii, Oahu I., Honolulu Co., A. A. Heller 2321 (holotype MIN!).

Iconography. Rúgolo de Agrasar (1974: 127); Vega \& Rúgolo de Agrasar (2012: 308).

Distribution. Widespread in tropical and subtropical regions of the world. USA, Mexico, Central America, the Caribbean, and South America (Colombia, Venezuela, Ecuador, Peru, French Guiana, Guyana, Surinam, Brazil, Paraguay, and Argentina).

Distribution and habitat in Puerto Rico and the Virgin Islands. Adjuntas, Cayey, Jayuya, Manatí, Maricao, Mayagüez, Ponce, Río Grande, and Sabana Grande. 1-1340 m.

Observations. $f l \& f r$ throughout the year.

\section{Additional specimens examined}

PUERTO RICO. Adjuntas. Barrio Limaní, off Rt. 525, Estación Agrícola, $550 \mathrm{~m}, 8 \mathrm{Feb}$ 2001, Axelrod \& Montalvo 11405 (UPRRP).

Cayey. Carite Forest Reserve, along road (off Rt 179) dividing Barrio Guavate from Barrio Farallón, 750-800 m, Axelrod \& Axelrod 4349 (UPRRP, US); Sierra de Cayey, Carite Forest Reserve, road 184, km 21.8-21.9, ca. $800 \mathrm{~m}$, Proctor \& Milety 43960 (SJ, US). Jayuya. Cerro de Punta (Highest Point in P.R.), $1338 \mathrm{~m}$, Gould et al. 15852 (US). Manatí. Tortuguero, Woodbury s.n. (P, UPRRP, US); Tierras Saliente, sandy area $\mathrm{S}$ of Laguna Tortuguero, $1 \mathrm{~m}$, Axelrod \& Comas 7488 (MO, UPRRP). Maricao. Maricao State Forest, beside office of Recreation Area, Proctor \& McKenzie 43828 (US). Mayagüez. Mayagüez, back of Exp. Station, Chase 6255 (US). Ponce. Toro Negro Forest Reserve, Rt 143, km 18.7, along dirt road to S slope of Monte Jajuya, ca. $1250 \mathrm{~m}$, Axelrod \& Chavez 4284 (UPRRP, US). Río Grande. Sierra de Luquillo, Caribbean National Forest, Roca El Yunque, ca. 1030 m, Proctor 42852 (SJ). Sabana Grande. Maricao State Forest, beside office of Recreation Area, ca. $850 \mathrm{~m}$, Proctor \& McKenzie 43828 (SJ, US).

\section{Excluded species}

Digitaria ekmanii Hitchc., Man. Grasses W. Ind. 243: 176. 1936.

This species was reported in Río Abajo (Puerto Rico) on the basis of the specimen AcevedoRodríguez 10578 (US) [Acevedo-Rodríguez \& Axelrod, 1999] and then excluded by Axelrod (2011) as a result of a misidentification. The name given for this specimen is Axonopus compressus (Sw.) P. Beauv.

Digitaria fuscescens (J. Presl) Henrard, Meded. Rijks-Herb. 61: 8. 1930. Paspalum fuscescens J. Presl, Reliq. Haenk. 1 (4-5): 213. 1830. TYPE: "Hab. in regione montana Peruviae" changed to "Hab. ad Monte-Rey Californiae" (on p. 351), T. P. Haenke s.n. (holotype PR; isotype $\mathrm{BM} !)$.

This species was introduced in Belize, the Caribbean, Colombia, Venezuela, Guyana, Surinam, French Guiana, Ecuador, Peru, Brazil, Bolivia, and Paraguay. In Puerto Rico was originally introduced from Jamaica [Alberts 274 (US)] and probably cultivated at the Río Piedras Experimental Station where Alberts did studies on forage grasses.

Digitaria panicea (Sw.) Urb., Symb. Antill. 8: 23. 1920. Milium paniceum Sw., Prodr. 24. 1788. TYPE: Jamaica, O. Swartz s.n. (holotype S photo!). 
This species was distributed in the Caribbean, Guatemala, Honduras, Mexico, and Panama, according to references cited in Vega \& Rúgolo de Agrasar (2003). Originally described from Jamaica and cited in continental Central America, although Henrard (1950) excluded this taxon from this area. This criterion is shared by the present authors, due to the fact that no specimens of $D$. panicea were found. Additionally, specimens from Cuba, Jamaica, Puerto Rico, and the Bermudas, identified by other authors as D. panicea, were misidentifications of Digitaria violascens, D. curvinervis, D. filiformis var. dolichophylla, D. argillacea var. nulliseta and D. ternata. Digitaria panicea is restricted to Jamaica and Cuba.

Digitaria $\times$ umfolozi D. W. Hall, Turrialba 33 (3): 327. 1983.

This taxon is a hybrid bred at the University of Florida (USA): Digitaria setivalva Stent $ᄋ \times$ D. valida Stent $\widehat{~}$ (Hall \& Schank, 1983), similar to Digitaria eriantha Steud. Many exotic species like this one were brought in and cultivated on farms in western Puerto Rico [Proctor \& GarcíaMolinari 46444 (SJ, US)]. They never really became naturalized.

\section{ACKNOWLEDGEMENTS}

The authors are especially grateful to curators of the herbaria for making the material available as well as V. Dudás for the illustration of the species. Also grateful to anonymous reviewers for their valuable suggestions. This work received financial support through grant PICT 2015-1472.

\section{BIBLIOGRAPHY}

Acevedo-Rodríguez, P. \& F. S. Axelrod. 1999. Annotated checklist for the Tracheophytes of Río Abajo Forest Reserve, Puerto Rico. Caribbean Journal of Science 35 (34): 265-285.

Axelrod, F. S. 2011. A systematic vademecum to the vascular plants of Puerto Rico. Sida, Bot. Misc. 34.
Britton, N. \& P. Wilson. 1923. Botany of Puerto Rico and the Virgin Islands. Scientific Survey of Puerto Rico and the Virgin Islands 5 (1): 121-130.

Canto-Dorow, T. S. \& H. M. Longhi-Wagner. 2001. Novidades taxonômicas em Digitaria Haller (Poaceae) e novas citações para o gênero no Brasil. Insula (Florianópolis) 30: 21-34.

Canto-Dorow, T. S. 2014. Digitaria in Lista de Espécies da Flora do Brasil. Jardim Botânico do Rio de Janeiro. Available at: http://reflora.jbrj.gov.br/jabot/floradobrasil/ FB13174 [accesed 27 Apr 2018].

Giraldo-Cañas, D. 2005. Las especies colombianas del género Digitaria (Poaceae: Panicoideae: Paniceae). Caldasia 27 (1): 25-87.

Giraldo-Cañas, D. 2016. Estudios morfológicos y taxonómicos en Digitaria Haller (Poaceae: Panicoideae: Paniceae): inventario y primer registro de Digitaria velutina (Forssk.) P. Beauv. para Sudamérica. Biota Colombiana 17(2): 1938. http://www.redalyc.org/articulo.oa? $\mathrm{id}=49151352003$ [accesed Nov. 2018].

Gould, F. W. 1979. Poaceae, in R. A. Howard (ed.), Flora of the Lesser Antilles, Leeward and Windward Islands 3: 25-220.

Hall, D. W. \& S. C. Schank. 1983. A name for University of Florida hybrid digitgrass x46-2. Turrialba 33 (3): 327-328.

Henrard, J. T. 1950. Monograph of the Genus Digitaria, Leiden: Universitaire Pers Leiden.

Hitchcock, A. S. 1936. Manual of the grasses of the West Indies. Washington: United States Government Printing Office.

Kok, P. D. F. 1984. Studies on Digitaria (Poaceae) I. Enumeration of species and synonymy. South African Journal of Botany 3 (3): 184-185.

Liogier, H. \& L. Martorell. 1982. Flora of Puerto Rico and adjacent islands: a systematic synopsis. Río Piedras: Editorial de la Universidad de Puerto Rico.

Liogier, H. A. \& L. F. Martorell. 2000. Flora of Puerto Rico and the adjacent islands: a systematic synopsis. $2^{\text {nd }}$ ed. Río Piedras: Editorial de la Universidad de Puerto Rico.

Lo Medico, J. M.; D. S. Tosto, G. H. Rua, Z. E. Rúgolo de Agrasar, M. A. Scataglini \& A. S. Vega. 2017. Phylogeny of Digitaria Sections Trichachne and Trichophorae (Poaceae, Panicoideae, Paniceae): A Morphological and Molecular Analysis. New Circumscription and Synopsis. Systematic Botany 42: 37-53. DOI: 10.1600/036364417X694908

Pohl, R. 1980. Gramineae, in W. Burger (ed.), Fieldiana, Botanical New Series No4. Flora Costaricensis, pp. 1-608. Chicago: Field Museum of Natural History.

Renvoize, S. A. 1998. Gramíneas de Bolivia. Kew: Kew Royal Botanic Gardens. 
Renvoize, S. A.; A. S. Vega \& Z. E. Rúgolo de Agrasar. 2006. Gramineae (part 3). Subfam. Panicoideae, in G. Harling \& C. Persson (eds.), Flora of Ecuador vol 78, pp. 1-216. Göteborg: Dept. of Plant and Environmental Sciences, University of Göteborg.

Rúgolo de Agrasar, Z. E. 1974. Las especies del género Digitaria (Gramineae) de la Argentina. Darwiniana 19: 65-166.

Rúgolo de Agrasar, Z. E. 1994. Digitaria (Gramineae), in R. Spichiger \& L. Ramella (eds.), Flora del Paraguay. Graminae $V$ (23), Panicoideae-Paniceae, pp. 75-138. Ginebra: Conservatoire et Jardin Botaniques.

Rúgolo de Agrasar, Z. E. \& M. L. Puglia. 2004. Gramíneas Ornamentales, in J. A. Hurrell (ed.), Plantas de la Argentina, pp. 336. Buenos Aires: Literature of Latin America (L.O.L.A.).

Sánchez-Ken, J. G. 2012. A synopsis of Digitaria (Paniceae, Panicoideae, Poaceae) in Mexico, including the new species Digitaria michoacanensis. Acta Botanica Mexicana 101: 127-149. DOI: 10.21829/abm101.2012.28

Soreng, R. J., P. M. Peterson, K. Romaschenko, G. Davidse, J. K. Teisher, L. G. Clark, P. Barberá, L. J. Gillespie \& F. O. Zuloaga. 2017. A worldwide phylogenetic classification of the Poaceae (Gramineae) II: An update and a comparison of two 2015 classifications. Journal of Systematics and Evolution 55: 259-290.

Thiers, B. (2018, continuously updated) Index Herbariorum: A global directory of public herbaria and associated staff. New York Botanical Garden's Virtual Herbarium. http:// sweetgum.nybg.org/science/ih/

UPRRP database project. 2018. Published on the Internet: http://136.145.224.102/Bol/uprrp/Search/RunSearch/ 34667?view=summary [accessed 10 May 2018].

Vega, A. S. \& Z. E. Rúgolo de Agrasar. 2001. Morphological interpretation of the spikelet in Digitaria atra (Poaceae: Panicoideae: Paniceae) and emended generic description. American Journal of Botany 88: 1670-1674. DOI: $10.2307 / 3558412$
Vega, A. S. \& Z. E. Rúgolo de Agrasar. 2002. Novedades taxonómicas y sinopsis del género Digitaria (Poaceae: Panicoideae: Paniceae) en Bolivia. Darwiniana 40: 171-190.

Vega, A. S. \& Z. E. Rúgolo de Agrasar. 2003. Digitaria, in F. O. Zuloaga, O, Morrone, G. Davidse, T. S. Filgueiras, P. M.Peterson, R. J. Soreng \& E. J. Judziewicz (eds.), Catalogue of New World Grasses (Poaceae): III. Subfamilies Panicoideae, Aristidoideae, Arundinoideae, and Danthonioideae. Contributions from the United States National Herbarium 46: 193-213.

Vega, A. S. \& Z. E. Rúgolo de Agrasar. 2005. Novedades taxonómicas y sinopsis del género Digitaria (Poaceae: Panicoideae: Paniceae) en Colombia y Venezuela. Darwiniana 43: 232-267.

Vega, A. S. \& Z. E. Rúgolo de Agrasar. 2006. Digitaria, in A. M. Molina \& Z. E. Rúgolo de Agrasar (eds.), Flora Chaqueña (Formosa, Chaco y Santiago del Estero): Familia Gramíneas. Colección Cientifica del Instituto Nacional de Tecnología Agropecuaria 23: 454-492.

Vega, A. S. \& Z. E. Rúgolo de Agrasar. 2007. Novedades taxonómicas y sinopsis del género Digitaria (Poaceae: Panicoideae: Paniceae) en América Central. Darwiniana 45: 92-119.

Vega, A. S. \& Z. E. Rúgolo de Agrasar. 2009. Digitaria, in R. Kiesling (ed.), Flora de San Juan IV República Argentina, pp. 257-262. Mendoza: Editorial Fundación Universidad Nacional de San Juan.

Vega, A. S. \& Z. E. Rúgolo de Agrasar. 2012. Digitaria, in F. O. Zuloaga, Z. E. Rúgolo de Agrasar \& A. M. Anton (eds.), Flora Argentina. Plantas Vasculares de la República Argentina. Monocotyledoneae: Poaceae: Aristidoideae-Pharoideae 3 (1): 287-308. Córdoba. Graficamente Ediciones.

Vega, A. S. \& Z. E. Rúgolo de Agrasar. 2014. Digitaria, in P. M. Jørgensen, M. H. Nee, \& S. G. Beck (eds.), Catálogo de las Plantas Vasculares de Bolivia. Monographs in Systematic Botany from the Missouri Botanical Garden 127 (2): 1056-1058.

Veldkamp, J. F. 1973. A revision of Digitaria Haller (Gramineae) in Malesia. Blumea 21: 1-80. 